\title{
Energy Storage Models for Optimizing Renewable Power Applications
}

\author{
${ }^{1}$ M.S. Okundamiya and ${ }^{2}$ A.N. Nzeako \\ ${ }^{1}$ Department of Electrical and Electronic Engineering, \\ Ambrose Alli University, P.M.B. 14, Ekpoma, Nigeria \\ ${ }^{2}$ University of Nigeria, Nsukka, Enugu State, Nigeria
}

\begin{abstract}
A detailed analysis of two well-known analytical models, the kinetic battery model and the diffusion model is presented which reveals that the kinetic battery model is a first-order approximation of the more complex diffusion model; both models are well suited for battery life time predictions. In order to optimize their storage capacity, super capacitors are introduced to the renewable energy system which creates a voltage source control that dictates the frequency and $\mathrm{AC}$ voltage in the system and mainly responds to sudden energy unbalances while a current source control receives control input from SC voltage difference and mitigates the error-signal in order to fulfill the necessary energy demand on the system.
\end{abstract}

Key words: Battery models, energy optimization, charging and discharging currents, state of charge, renewable energy system, control strategies, super capacitors

\section{INTRODUCTION}

Under the pressure of limited available energy resources and environmental policies, electrical power generation has rapidly increased in recent years. When the load demand of the system is less than the output of the generation of the renewable energy, the excess electric power needs to be stored in an efficient energy storage system to supply the load during calm periods. Due to the nature of intermittence of renewable energy, the use of energy storage such as batteries become inevitable which will compensate the fluctuations of power when there is shortage of generation. A battery is a widely known component that converts electrical energy into chemical energy and vice versa. In Lead- acid batteries, the charge process leads to a reform of the tetraoxosulphate (vi) acid of the electrolyte as:

$$
\mathrm{SO}_{4}^{2-}+2 \mathrm{H}^{+} \rightarrow \mathrm{H}_{2} \mathrm{SO}_{4}
$$

The net (re) charge reaction on both plates and electrolyte is:

$$
2 \mathrm{PbSO}_{4}+2 \mathrm{H}_{2} \mathrm{O} \rightleftharpoons \mathrm{PbO}_{2}+2 \mathrm{H}_{2} \mathrm{SO}_{4}+\mathrm{Pb}
$$

Batteries may be required to supply long-duration energy-type load or short-duration power-type loads, depending on their applications. Any battery can be sized to supply short-duration power loads but high-power battery designs supply them more effectively than others. The life time of batteries depends naturally on the rate at which energy is consumed and the usage pattern of the battery as frequent charging and discharging will shorten the life time of a battery. Continuous drawing of a high current results in an excessive drop of residual capacity. However, during intervals with no or very small currents, batteries do recover to a certain extent.

The optimal charging curve of the Lead-acid battery based on the lowest output gas rate is shown in Fig. 1 (Oneyama, 1998). If the charging current keeps to the track of the optimal curve, the charging hours can be sharply cut down wihout any side effect on the capacity and lifetimes of the battery. The charging current and time of the Lead-acid battery is related by (Zhao, 2006):

$$
\mathrm{i}_{\mathrm{c}}=\mathrm{I}_{\mathrm{o}} \mathrm{e}^{-\alpha t}
$$

Where:

$i_{c}=$ The value of the instanteneous charging current in each moment

$1_{0}=$ The initial charging current which is the maximum current value in the whole process

$\alpha=$ The attenuation coefficient of the battery

The Ampere-hour rule for charging the Lead-acid batteries can be considered as the most efficient charging approach, considering the charging time and the excellent performance provided by this method to maintain the lifetime of the battery (Yin et al., 2008).

Corresponding Author: M.S. Okundamiya, Department of Electrical and Electronic Engineering, Ambrose Alli University, P.M.B. 14, Ekpoma, Nigeria 
Int. J. Elec. Power Eng., 4 (2): 54-65, 2010

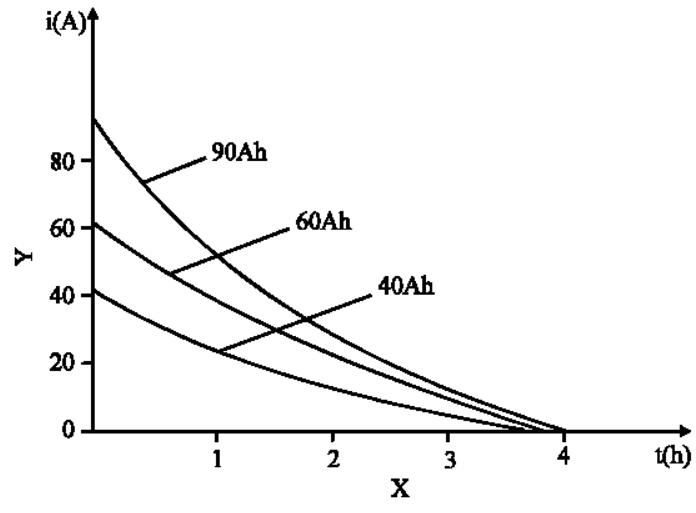

Fig. 1: Optimal charging curve of lead-acid battery

When the battery is charging, the charging current $I_{c}$, the charging voltage $V_{c}$, the charging potential difference $\mathrm{E}_{\mathrm{b}}$ between the positive plate and negative plate of the battery and the internal resistor $\mathrm{R}_{b}$ of the battery has the relationship:

$$
I_{c}=\frac{V_{c}-E_{b}}{R_{b}}
$$

Thus the battery can only be charged when $V_{c}>E_{b}$ and $E_{b}$ changes with the charging process. The control system of battery charging and discharge process is a non-linear, time-varying system with pure time delay, multiple variables and many outer disturbances.

Such parameters as charging rate, permitted maximum charging current, internal resistor, port voltage, temperature, moisture and life time, etc., vary with different battery.

Indeed, it has been observed recently that due to the specific physical nature of batteries, achieving the longest battery lifetime is not always achieved by trying to minimize only the power consumption at any point in time. Instead, also the way in which the power is consumed that is the current-extraction patterns and the employed current levels play a role in battery lifetime. The energy consumption rates of a system can be interpreted as a Markov-reward model in which accumulated reward stands for the amount of energy consumed. The system or battery lifetime then equals the time until a certain level of consumption (the available charge of the battery) is reached.

The time distribution can be done with well known techniques for performability evaluation (Cloth et al., 2007), however such an approach does not accurately take into account the physical aspect of battery operation. Studies on batteries reveal that the battery depletion rate in general is non-linear in time which depends on the amount of energy still in the battery (Cloth et al., 2007;
Jongerden and Haverkort, 2008). In periods when a battery is not used, subtle but important batteryrestoration effects are in place that apparently refill the battery.

To capture the influence of the power consumption on the battery, a battery model is needed. Many different types of battery models have been developed for different application areas (Jongerden and Haverkort, 2008). The electro-chemical models (Doyle et al., 1993; Fuller et al., 1994) for battery design, describe the battery in a very detailed manner using a set of six coupled differential equations while the electrical circuit models used in electrical engineering (Hageman, 1993) focus on the electrical properties of the battery. These models are not suitable to be used in the setting of the performance models because of the detailed description which would make the combined model unmanageable, thus an abstract model which focuses on the important battery properties and their effects only is needed.

Two analytical models: the Kinetic Battery Model (KiBaM) (Manwell and McGowan, 1993; Manwell et al., 1994) and the diffusion based model (Rakhmatov and Vrudhula, 2001) describe the battery using only two differential equations. Theoretical comparison of both models is made which leads to the conclusion on which model is best to use in setting of the performance models.

General overview of the renewable energy system: Figure 2 shows the schematics of a renewable energy system. With such a renewable energy system, the problem is how to determine when the battery bank should be charged to provide the best energy efficiency and to prolong the lifetime. The battery bank works in three statuses:

- $\quad$ Charged by the Renewable Energy System (RES)

- Discharged to supply the load

- Disconnected from the RES

The status of the battery is dependent on the working modes and shifts according to the different modes of the system. A common issue to be dealt with in the design is power consumption and a key issue to be addressed is to find the right tradeoff between battery usage and required performance: how should the processes in the RES be organised such that the battery lifetime (which determines the system lifetime) will be as high as possible.

Maintaining energy and power balance, especially when the size of the system is small is a major problem in most RES, since sharp fluctuations negatively affect battery lifetime. In this study, super capacitors are introduced to responds to sudden energy unbalances. 


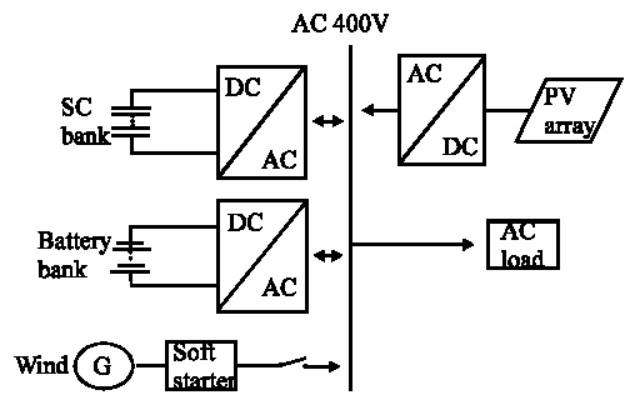

Fig. 2: Schematics of a renewable energy system

The DC/AC converter connected to the SC bank acts as a Voltage Source Converter (VSC) while the DC/AC converter connected to the battery-bank acts as a Current Source Converter CSC. The VSC is the primary storage of the RES. It dictates the frequency and $\mathrm{AC}$ voltage in the system and mainly responds to sudden energy unbalances. The $\mathrm{CSC}$ receives control input from $\mathrm{SC}$ voltage difference and mitigates the error-signal in order to fulfill the necessary energy demand of the system.

Analysis of battery models: The usage pattern of a device can be well modeled with stochastic workload models. However, one still needs a battery model to describe the effects of the power consumption on the state of the battery. Over the years, many different types of battery models have been developed for different application areas. There are many types of batteries and many factors that affect battery performance. To predict the performance of a battery, many different mathematical models exist. None of these models are completely accurate nor do any include all the necessary performance-effecting factors. Factors that affect battery performance include:

- $\quad$ State of Charge (SOC)

- Rate of charge/discharge

- Battery storage capacity

- Temperature

- Age/shelf life

The essential battery properties need to be modeled to obtain an accurate battery model. The two most important properties of a battery are its voltage, $\mathrm{V}$ and its capacity, Ah; the product of these two quantities is a measure of the energy stored in the battery. For an ideal battery the voltage stays constant over time until the moment it is completely discharged then the voltage drops to zero. The capacity in the ideal case is the same for every load on the battery. In reality due to rate capacity effect the effective perceived capacity is lower under higher load while voltage drops during discharge. In the ideal case the lifetime, $\mathrm{L}$ of a battery with a constant load of capacity, C over the load current, I is:

$$
\mathrm{L}=\frac{\mathrm{C}}{\mathrm{I}}
$$

Due to various non-linear effects, a simple approximation for the lifetime of real battery under constant load is obtained by Peukert's law Eq. 11 as:

$$
\mathrm{L}=\frac{\mathrm{a}}{\mathrm{I}^{\mathrm{b}}}
$$

where $a>0$ and $b>1$ are constants which depend on the battery. For variable loads, $i(t)$ the sum of average current until $\mathrm{t}=\mathrm{L}$ in Eq. 6 becomes:

$$
L=\frac{a}{\left(\frac{1}{L} \int_{0}^{L} i(t) d t\right)^{b}}
$$

Thus all load profiles with the same average current have the same lifetime but due to recovery effect of the battery, i.e., the effect that the battery regains some of its lost capacity during idle periods, the results of Eq. 7 deviate from real values.

Electrochemical models: The electrochemical models are the most accurate battery models since they are based on the chemical processes that take place in the battery which describe these battery processes in great detail. However, the highly detailed description makes the models complex and difficult to configure.

The simplest electrochemical models are solely based on electrochemistry while these models can predict energy storage they are unable to model phenomema such as time rate of change of voltage under load and they ignore thermodynamic and quatum effects. The state of charge, $\mathrm{SOC}$ at a constant discharge rate, $\mathrm{C}$ is:

$$
\mathrm{SOC}=1-\frac{\mathrm{It}}{\mathrm{C}}
$$

For non-constant discharge rates:

$$
\delta \mathrm{SOC}=\frac{\mathrm{I}_{2} \Delta \mathrm{t}}{3600 \mathrm{C}_{1}} \cdot\left(\frac{\mathrm{I}_{2}}{\mathrm{I}_{1}}\right)^{\mathrm{N}-1}
$$

Where:

$$
\mathrm{C}_{1}=\mathrm{C}_{2}\left(\frac{\mathrm{I}_{2}}{\mathrm{I}_{1}}\right)^{\mathrm{N}-1}
$$


The battery constant $\mathrm{N}$, varies between 1.1 and 1.2 for a typical lead-acid battery. The shepherd model which tries to find the cutoff point beyond which the terminal voltage decreases very rapidly describes the electrochemical behaviour of the battery directly in terms of voltage and current and it is often used in conjunction with Peukert equation to obtain battery voltage and SOC given power draw variations while Wood model incorporates secondary equation to describe overcharging and gas generation, along with a selfdischarge term (Moore and Merhdad, 1996; Unnewehr and Nasar, 1982). A simplifying shepherd's (Unnewehr and Nasar, 1982) attempts to model the variation in the internal resistance, $\mathrm{R}_{\mathrm{i}}$ with respect to $\mathrm{SOC}$ creating the charge and dischare currents. Dualfoil is a Fortran program that uses the electrochemical model to simulate lithium-ion batteries. The program is freely available on the internet. It computes how all the battery properties change over time for the load profile set by the user. From the output data, it is possible to obtain the battery lifetime. Besides the load profile, the user has to set over 50 battery related parameters, e.g., the thickness of the electrodes, the initial salt concentration in the electrolyte and the overall heat capacity etc.

To be able to set all these parameters one needs a very detailed knowledge of the battery that is to be modeled. On the other hand, the accuracy of the program is very high. The program is often used as a comparison against other models, instead of using experimental results to check the accuracy.

Equivalent-circuit models: The charge storing capacity of the battery is often represented by a capacitor such as the Kleckner (discharge-only) and the Zimmerman-Peterson models (Moore and Merhdad, 1996). Other models employ a capacitor in parallel with the steady-state battery (voltage source in series with a resistor representing the internal resistance) to limit the rate of change of the battery's voltage which the steady state model would not predict (Dobner and Woods, 1982).

In such models, the total current is the sum of the steady state current and the capacitor current. The steady state battery voltage as a function of staedy state current is obtained from tabulation of measured data while the capacitor current is:

$$
I_{c}=C \cdot \frac{d V}{d t}
$$

where $\mathrm{C}$ is the capacitance of the capacitor.

Electrical-circuit models: In electrical-circuit models the electrical properties of the battery are modeled using

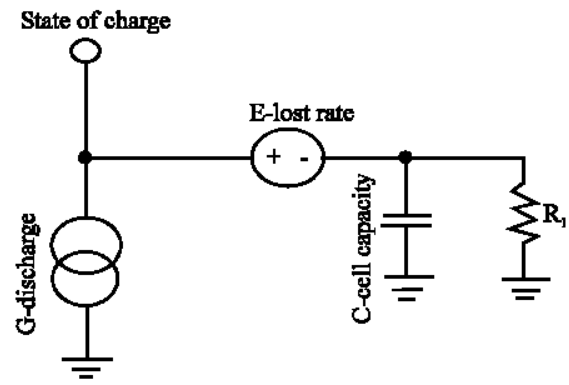

Fig. 3: Basic circuit model of an arbitrary cell

PSpice circuits consisting of voltage sources, lookup tables and linear passive elements, such as resistors and capacitors. The electrical-circuit models proposed by Hageman (1993) uses simple PSpice circuits to simulate Nickel-Cadmium, Lead-acid and alkaline batteries. The core of the different types of batteries of the electricalcircuit models are as follows:

- Capacitor represents the capacity of the battery

- Discharge-rate normaliser determines the lost capacity at high discharge currents

- Circuit to discharge the capacity of the battery

- Voltage versus state-of-charge lookup table

- Resistor representing the battery's resistance

Figure 3 shows the basic circuit model of an arbitrary cell. Minor changes have to be made to completely model a specific cell type. Although, the model is simpler than the electrochemical model and therefore computationally less expensive, it still takes quite some effort to configure them.

Especially, the lookup tables used in the model require much experimental data on the battery's behaviour with less accurate in predicting battery lifetime, having errors up to approximately $12 \%$ (Gold, 1997).

Temperature model: The impact that temperature exerts on battery's capacity can be explained with a simple model of the battery's electrochemistry. As the temperature increases towards the peak-performanceoperating temperature the electrolytic viscosity decreases, thus allowing increased diffusion of ions and hence increased battery's capacity. When the temperature exceeds the peak point the battery begins to corrode which reduces the active electrode area resulting in fewer electrode reaction and a reduced capacity. This impact starts with the general equation for battery capacity (Roan and Anand, 1993):

$$
\mathrm{C}=\mathrm{C}_{25}(1-\alpha(25-\mathrm{t}))
$$



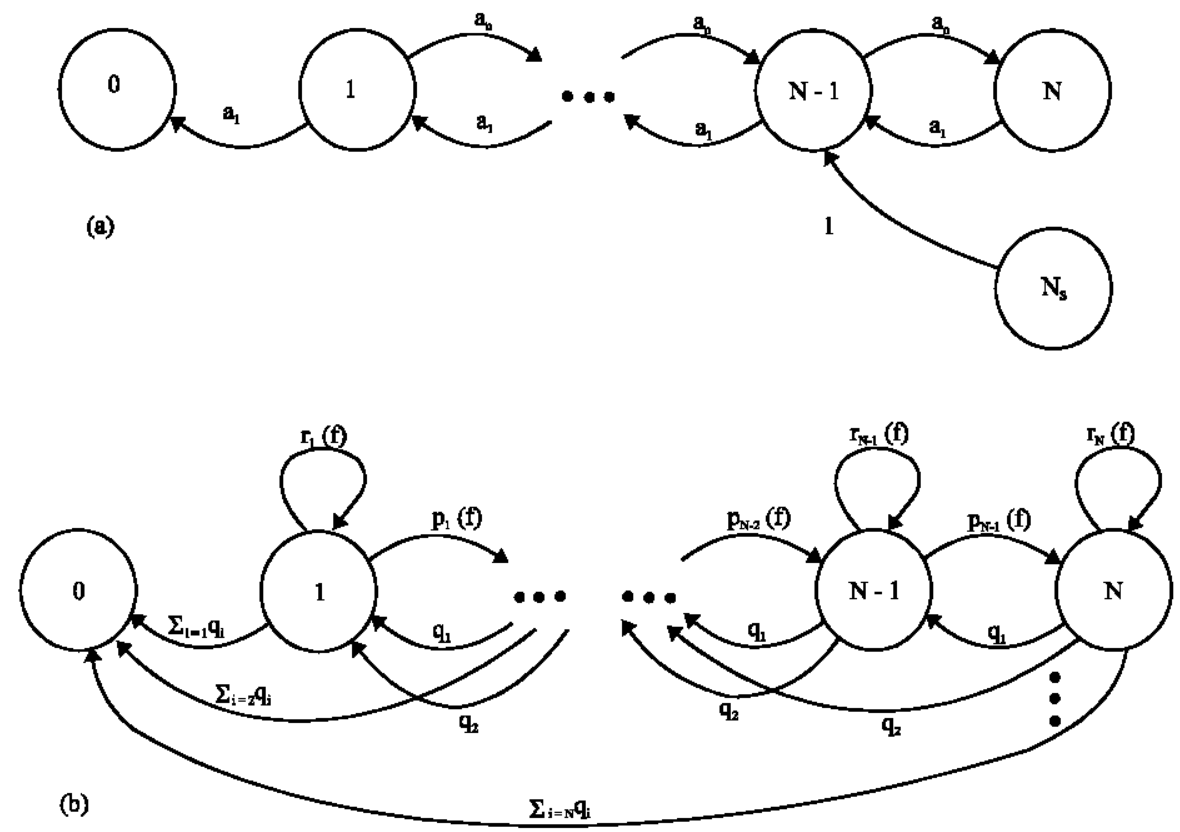

Fig. 4: State transition diagram of Markov chain battery model: (a) basic (b) extended

Where:

$\mathrm{C}=$ Battery's capacity at temperature, $\mathrm{T}(\mathrm{Ah})$

$\mathrm{C}_{25}=$ Battery's capacity at $25^{\circ} \mathrm{C}(\mathrm{Ah})$

$\alpha=$ Temperature coefficient, $\left(\mathrm{Ah} /{ }^{\circ} \mathrm{C}\right)$

$\mathrm{T}=$ The battery's temperature, $\left({ }^{\circ} \mathrm{C}\right)$

The temperature coefficient which varies from battery to battery is experimentally determined, typically formulated as a quadratic function of temperature.

Stochastic models: Figure 4 shows the stochastic models developed by Chiasserini and Rao (2001 a) which describe the battery at a higher level of abstraction than the electrochemical and electrical-circuit models (Chiasserini and Rao, 1999). It describes two models of a battery of a mobile communication device for transmitting packets.

The basic model shown in Fig. 4a, describes the battery by a discrete time Markov chain with $\mathrm{N}+1$ states, numbered from $0-\mathrm{N}$ which corresponds to the number of charge units available in the battery. One charge unit corresponds to the amount of energy required to transmit a single packet.

$\mathrm{N}$ is the number of charge units directly available based on continuous use. The time step is either a charge unit is consumed with probability $a_{1}=q$ or recovery of one unit of charge takes place with probability $a_{0}=1-q$. The battery is considered empty when the absorbing state 0 is reached or when a maximum of $\mathrm{T}$ charge units have been consumed.
The number of charge units is equal to the theoretical capacity of the battery $(\mathrm{T}>\mathrm{N})$. The rate of recovery is not constant during discharge and in most systems the discharge current changes over time.

The extended Markov chain battery model of Fig. 4b was developed to overcome the limitations of the basic model, incorporating several extensions (Chiasserini and Rao, 2001 a, b).

The recovery probability is made state dependent thus when less charge units are available the probability to recover a charge unit will become smaller. Due to phase dependence the probability of recovery decrease with increase in phase number. The non-zero probability of staying in the same state in which no energy consumption or recovery takes place during a time step is also included.

During the idle periods, the battery either recovers one charge unit with probability $P_{j}(f)$ or stays in the same state with probability $r_{j}(f)$. The recovery probability in state $j$ and phase $f$ is defined as (Chiasserini and Rao, 2001b):

$$
P_{j}(f)=q_{0} e^{(\mathbb{N - j )}) \mathrm{N}-\mathrm{g}_{\mathrm{c}}(\mathrm{f})}
$$

Where $g_{N}$ and $g_{c}$ (f) depend on the recovery behaviour of the battery. These results show that the stochastic model gives a good qualitative description of battery behaviour under pulsed discharge. However, it is 
unclear how well the model performs quantitatively, since only results of the gain and no numbers for the computed lifetimes are given.

Analytical models: The two analytical battery models discussed; Kinetic Battery Model and diffusion model which describe the non-linear effects of the battery using two differential equations.

Kinetic battery model: The Kinetic Battery Model (KiBaM) of Manwell and McGowan (1993) and Manwell et al. (1994) is a very intuitive battery model that uses a chemical kinetics process as its basis. In the model, the battery charge is distributed over two wells: availablecharge well and bound-charge well; as shown in Fig. 5.

A fraction $\mathrm{c}$ of the total capacity is placed in the available charge well (denoted $\mathrm{y}_{1}(\mathrm{t})$ ) and a fraction 1 - $\mathrm{c}$ in the bound charge well (denoted $\left.y_{2}(t)\right)$. The available charge well supplies electrons directly to the load (i (t)), whereas the bound-charge well supplies electrons only to the available-charge well. The charge flows from the bound charge well to the available charge well through a valve with fixed conductance, $k$. The rate at which charge flows between the wells depends on the height difference between the two wells. The heights of the two wells are given by:

$$
\begin{gathered}
\mathrm{h}_{1}=\frac{\mathrm{y}_{1}}{\mathrm{c}} \\
\mathrm{h}_{2}=\frac{\mathrm{y}_{2}}{1-\mathrm{c}}
\end{gathered}
$$

From Fig. 5, the change of the charges in both wells are respectively given by Eq. 16 and 17 as:

$$
\begin{gathered}
\frac{d y_{1}}{d t}=-i(t)+k\left(h_{2}-h_{1}\right) \\
\frac{d y_{2}}{d t}=-k\left(h_{2}-h_{1}\right)
\end{gathered}
$$

with initial conditions $\mathrm{y}_{1}(0)$ and $\mathrm{c}(\mathrm{C})$ where $\mathrm{C}$ is the total battery capacity. The battery is considered empty when there is no charge left in the available charge well.

When a load is applied to the battery, the available charge reduces and the height difference between the two wells grows. When the load is removed, charge flows from the bound-charge well to the available-charge well until $h_{1}$ and $h_{2}$ are equal again. So, during an idle period, more charge becomes available and the battery lasts longer than when the load is applied continuously. In this way, the recovery effect is taken into account. Also, the rate

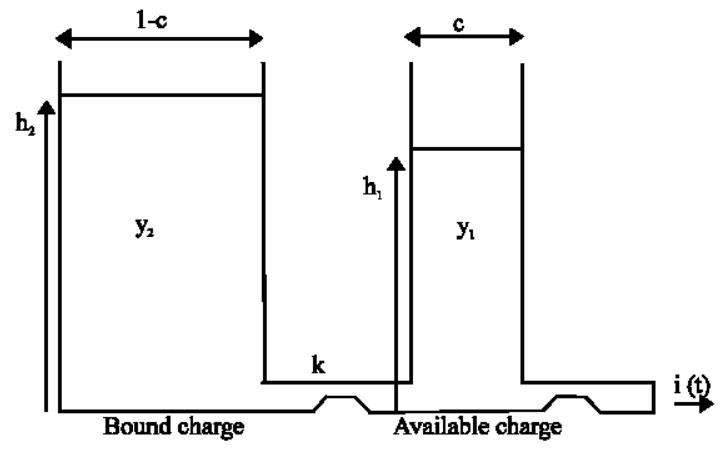

Fig. 5: A two-well Kinetic battery model

capacity effect is covered, since for a higher discharge current the available charge well will be drained faster and less time will be available for the bound charge to flow to the available charge. Therefore, more charge will remain unused and the effective capacity is lower.

Applying Laplace transforms to Eq. 16 and 17 for constant discharge current, $\mathrm{I}(\mathrm{t})=1$ yields:

$$
\begin{gathered}
\mathrm{y}_{1}(\mathrm{t})=\mathrm{y}_{1,0} \mathrm{e}^{-\mathrm{k}^{\prime} \mathrm{t}}+\frac{\left(\mathrm{y}_{0} \mathrm{k}^{\prime} \mathrm{c}-1\right)\left(1-\mathrm{e}-\mathrm{k}^{\prime} \mathrm{t}\right)}{\mathrm{k}^{\prime}}+ \\
\frac{\mathrm{Ic}\left(\mathrm{k}^{\prime} \mathrm{t}-1+\mathrm{e}^{-\mathrm{k}^{\prime} \mathrm{t}}\right)}{\mathrm{k}^{\prime}} \\
\mathrm{y}_{2}(\mathrm{t})=\mathrm{y}_{2,0} \mathrm{e}^{-\mathrm{k}^{\prime} t}+\mathrm{y}_{0}(1-\mathrm{c})\left(1-\mathrm{e}^{-\mathrm{k}^{\prime} \mathrm{t}}\right)+ \\
\frac{\mathrm{I}(1-\mathrm{c})\left(\mathrm{k}^{\prime} \mathrm{t}-1+\mathrm{e}^{-\mathrm{k}^{\prime} \mathrm{t}}\right)}{\mathrm{k}^{\prime}}
\end{gathered}
$$

where, $\mathrm{y}_{1,0}$ and $\mathrm{y}_{2,0}$ are the amount of available and bound charge respectively, at $\mathrm{t}=0$;

$$
\begin{gathered}
\mathrm{k}^{\prime}=\mathrm{k} \frac{\mathrm{c}-1}{\mathrm{c}} \\
\mathrm{y}_{0}=\mathrm{y}_{1,0}+\mathrm{y}_{2,0}
\end{gathered}
$$

The height difference between the two wells $\left(h_{2}-h_{1}\right)$ of Fig. 4 and the total charge, $y_{1}+y_{2}$ play a major role in the model. Transforming these coordinates to:

$$
\begin{gathered}
\delta=\left(\mathrm{h}_{2}-\mathrm{h}_{1}\right) \\
\gamma=\left(\mathrm{y}_{1}+\mathrm{y}_{2}\right)
\end{gathered}
$$

Applying these transformation changes to Eq. 16 and 17 give:

$$
\frac{\mathrm{d} \delta}{\mathrm{dt}}=\frac{\mathrm{i}(\mathrm{t})}{\mathrm{c}}-\mathrm{k}^{\prime} \delta
$$




$$
\frac{\mathrm{d} \gamma}{\mathrm{dt}}=-\mathrm{i}(\mathrm{t})
$$

with initial conditions $\delta(0)=0$ and $\gamma(0)=\mathrm{c}$. The condition for the battery to be empty is:

$$
\gamma(\mathrm{t})=(1-\mathrm{c}) \delta(\mathrm{t})
$$

Applying Laplace transforms to Eq. 24 and 25 for constant discharge current, $i(t)=1$ yields:

$$
\begin{gathered}
\delta(\mathrm{t})=\frac{1}{\mathrm{c}} \cdot \frac{1-\mathrm{e}^{-\mathrm{k}^{\prime} \mathrm{t}}}{\mathrm{k}^{\prime}} \\
\gamma(\mathrm{t})=\mathrm{C}-\mathrm{It}
\end{gathered}
$$

During idle periods the height difference will decrease due to the flow of charge from the bound charge well to the available charge well. The function that describes the evolution of the height difference during an idle period after a load I that lasted for a period of length $t_{i}$ :

$$
\delta\left(\mathrm{t}_{\mathrm{i}}\right)=\frac{1}{\mathrm{c}} \cdot \frac{\mathrm{e}^{-\mathrm{k}^{\prime} \mathrm{t}_{\mathrm{i}}} 1-\mathrm{e}^{-\mathrm{k} \mathrm{k}_{\mathrm{i}}}}{\mathrm{k}^{\prime}}
$$

where, $t_{i}$ is the idle time. The solutions for continuous discharge can be used to obtain a solution for any discharge profile with piecewise constant currents by adapting the initial conditions appropriately. The level of $\gamma$ and $\delta$ at the end of a step in the load profile can then be used as initial conditions for the next step.
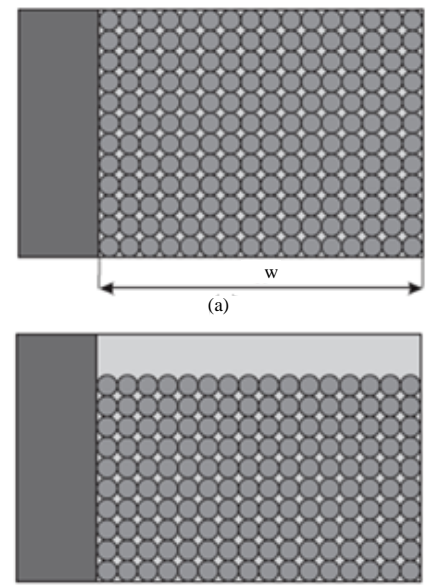

(c)
Diffusion model: The analytical battery model developed by Rakhmatov and Vrudhula (2001) is based on the diffusion of the ions in the electrolyte (Chiasserini and Rao, 2001a, b) which describes the evolution of the concentration of the electro-active species in the electrolyte to predict the battery lifetime under a given load. It assumes that the processes at both electrodes are identical, thus the battery is symmetrical with respect to the electrodes and only one of the electrodes is considered. Figure 6 shows a simplified view of the operation of the diffusion model.

Figure 6a shows the concentration of the electroactive species of the full battery which is constant over the full width $(\mathrm{w})$ of the electrolyte. When a load is applied to the battery, the electrochemical reaction results in a reduction in the concentration of the species near the electrode that create a gradient across the electrolyte as shown in Fig. $6 b$.

This gradient causes the species to diffuse towards the electrode. When the load is switched off, the concentration of the species at the electrode will recover (increase again) due to diffusion and eventually the species will be evenly distributed over the electrolyte again with a lesser concentration as shown in Fig. 6c. Finally, when the concentration at the electrode drops below the cut off value $\left(\mathrm{C}_{\text {cutoff }}\right)$, the battery will no longer be able to substain the chemical reaction hence, it is considered empty as shown in Fig. $6 \mathrm{~d}$.

The concentration of the electro-active species at time $t$ and distance $x \in(0, w)$ is denoted by $C(x, t)$. For the full battery, the concentration is constant over the length of the electrolyte. The battery is considered empty when

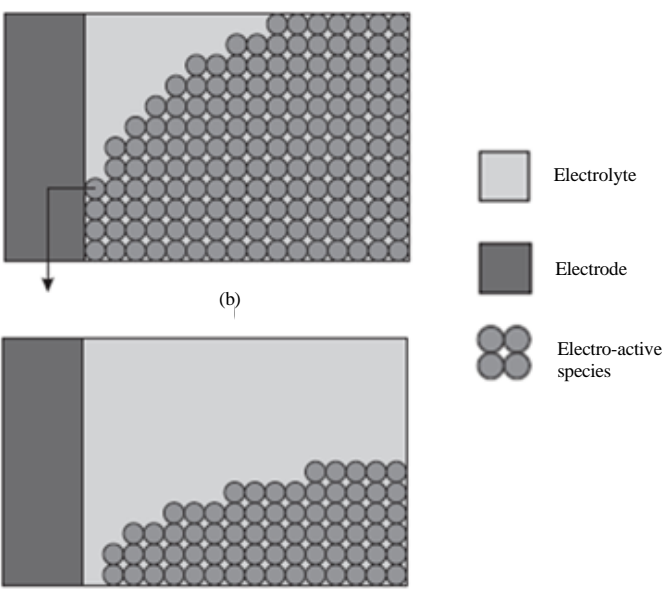

(d)

Fig. 6: Simplified view of the operation of the diffusion model (a) charged state (b) before recovery (c) after recovery (d) discharged state 
$\mathrm{C}(0, \mathrm{t})$ drops below the cutoff level $\left(\mathrm{C}_{\text {cutoff }}\right)$. The evolution of the concentration is described by Fick's laws (Rakhmatov and Vrudhula, 2001) as:

$$
\begin{aligned}
& -J(x, t)=D \frac{\partial C(x, t)}{\partial x} \\
& \frac{\partial C(x, t)}{\partial t}=D \frac{\partial^{2} C(x, t)}{\partial x^{2}}
\end{aligned}
$$

where, $J(x, t)$ is the flux of the electro-active species at time $\mathrm{t}$ and distance $\mathrm{x}$ from the electrode and $\mathrm{D}$ is the diffusion constant.

The flux at the electrode surface $(x=0)$ is proportional to the current $i(t)$. The flux on the other side of the diffusion region $(\mathrm{x}=\mathrm{w})$ equals zero. This leads to the boundary conditions:

$$
\begin{gathered}
\left.D \frac{\partial C(x, t)}{\partial x}\right|_{x=0}=\frac{i(t)}{v F A} \\
\left.D \frac{\partial C(x, t)}{\partial x}\right|_{x=w}=0
\end{gathered}
$$

Where:

$\mathrm{A}=$ The area of the electrode surface

$\mathrm{F}=$ Faraday's constant

$v=$ The number of electrons involved in the electrochemical reaction at the electrode surface

Applying Laplace transforms to Eq. 30 and 31 together with the initial and boundary conditions of Eq. 32 and 33 gives the analytical solution from which an expression for the apparent charge lost from the battery $\sigma(\mathrm{t})$ of Eq. 34 can be obtained (Rao et al., 2005).

$$
\sigma(t)=a(t)+u(t)
$$

The available charge supplied to the load, a ( $\mathrm{t}$ ) (being used by the device) is:

$$
\mathrm{a}(\mathrm{t})=\int_{0}^{\mathrm{t}} \mathrm{i}(\tau) \mathrm{d} \tau
$$

and the unavailable charge $\mathrm{u}(\mathrm{t})$ which remains unused in the battery is:

$$
\mathrm{u}(\mathrm{t})=\int_{0}^{\mathrm{t}} \mathrm{i}(\tau)\left(2 \sum_{\mathrm{m}=1}^{\infty} \mathrm{e}^{-\beta^{2} \mathrm{~m}^{2}(\mathrm{t}-\tau)}\right) \mathrm{d} \tau
$$

Where;

$$
\beta=\frac{\pi \sqrt{\mathrm{D}}}{\mathrm{W}}
$$

The battery is empty when the apparent available charge supplied is equal to the battery's capacity. For a constant current I, Eq. 35 reduces to:

$$
\mathrm{a}(\mathrm{t})=\mathrm{It}
$$

and Eq. 36 becomes:

$$
u(t)=2 I \sum_{m=1}^{\infty} \frac{1-e^{-\beta^{2} m^{2} t}}{\beta^{2} m^{2}}
$$

During idle periods the unavailable charge will decrease and will be available again for the load. The evolution of the unavailable charge during an idle period after a load I that lasted for a period of length $t_{1}$ is:

$$
u\left(t_{i}\right)=2 I \sum_{m=1}^{\infty} \frac{e^{-\beta^{2} m^{2} t_{i}}\left(1-e^{-\beta^{2} m^{2} t_{i}}\right)}{\beta^{2} m^{2}}
$$

where $t_{i}$ is the idle time.

Comparing KiBaM and diffusion model: In both models the charge in the battery has to flow to one side to be available for usage and part of it will stay behind in the battery when the battery is empty. To show that the diffusion model actually is a continuous version of the $\mathrm{KiBaM}$, we need to normalise the width (w) of the battery thus:

$$
\mathrm{x}^{\prime}=\frac{\mathrm{x}}{\mathrm{W}}
$$

where, $\mathrm{x}^{\prime}$ is a dimensionless space co-ordinate and takes a value between 0 and 1. Applying Eq. 41 to Eq. 30 and 31, we have:

$$
\begin{aligned}
& -J\left(x^{\prime}, t\right)=\frac{D}{w} \cdot \frac{\partial C\left(x^{\prime}, t\right)}{\partial x^{\prime}} \\
& \frac{\partial C\left(x^{\prime}, t\right)}{\partial t}=\frac{D}{w^{2}} \cdot \frac{\partial^{2} C\left(x^{\prime}, t\right)}{\partial x^{\prime 2}}
\end{aligned}
$$

and the boundary conditions:

$$
\begin{gathered}
\left.\frac{\mathrm{D}}{\mathrm{w}} \cdot \frac{\partial \mathrm{C}\left(\mathrm{x}^{\prime}, \mathrm{t}\right)}{\partial \mathrm{x}^{\prime}}\right|_{\mathrm{x}^{\prime}=0}=\frac{\mathrm{i}(\mathrm{t})}{v \mathrm{FA}} \\
\left.\frac{\mathrm{D}}{\mathrm{w}} \cdot \frac{\partial \mathrm{C}\left(\mathrm{x}^{\prime}, \mathrm{t}\right)}{\partial \mathrm{x}^{\prime}}\right|_{\mathrm{x}^{\prime}=1}=0
\end{gathered}
$$
thus:

Every ion yields $v$ electrons in the chemical reactions, 


$$
h\left(x^{\prime}, t\right)=C\left(x^{\prime}, t\right) v F A
$$

applying Eq. 46 to 42 through Eq. 45 yields:

$$
\begin{gathered}
-\mathrm{J}\left(\mathrm{x}^{\prime}, \mathrm{t}\right) v \mathrm{FA}=\frac{\mathrm{D}}{\mathrm{w}} \cdot \frac{\partial \mathrm{h}\left(\mathrm{x}^{\prime}, \mathrm{t}\right)}{\partial \mathrm{x}^{\prime}} \\
\frac{\partial \mathrm{h}\left(\mathrm{x}^{\prime}, \mathrm{t}\right)}{\partial \mathrm{t}^{\prime}}=\frac{\mathrm{D}}{\mathrm{w}^{2}} \cdot \frac{\partial^{2} \mathrm{y}\left(\mathrm{x}^{\prime}, \mathrm{t}\right)}{\partial \mathrm{x}^{\prime 2}} \\
\left.\frac{\mathrm{D}}{\mathrm{W}} \cdot \frac{\partial \mathrm{h}\left(\mathrm{x}^{\prime}, \mathrm{t}\right)}{\partial \mathrm{x}^{\prime}}\right|_{\mathrm{z}^{\prime}=0}=\mathrm{i}(\mathrm{t}) \\
\left.\frac{\mathrm{D}}{\mathrm{W}} \cdot \frac{\partial \mathrm{h}\left(\mathrm{x}^{\prime}, \mathrm{t}\right)}{\partial \mathrm{x}^{\prime}}\right|_{\mathrm{x}^{\prime}=1}=0
\end{gathered}
$$

Figure 7 shows a schematic view of the discretised model of the spatial co-ordinate $\mathrm{x}^{\prime}$. The electrolyte is divided in $\mathrm{n}$ parts of size $\alpha=1 / \mathrm{n}$.

The level of the charge in part $i$ is denoted by denotes $h_{i} h_{1}(t)$ while $h(0, t)$ denotes $h(1, t)$. Applying the finite difference method for second-order derivatives:

$$
\frac{\partial^{2} h}{\partial x^{2}}=\frac{h(x+a)-2 h(x)+h(x-\alpha)}{\alpha^{2}}
$$

where, $\alpha$ is the step-size of the discretisation. This results in a system of $n$ coupled differential equations:

$$
h_{n}-h_{n-1}
$$

Equation 52 is exactly the equation one would get when the KiBaM model is extended to $\mathrm{n}$ equally sized wells. The variable $h_{i}(t)$ gives the height of well number $i$ at time $t$. When $n=2$, the model is reduced to the KiBaM with two wells, each containing half of the total charge, that is $\mathrm{c}=0.5$ and $\mathrm{k}=2 \mathrm{D} / \mathrm{w}^{2}$.

The unavailable charge in KiBaM is $\left(h_{2}-h_{1}\right) \cdot(1-c)$ and for constant discharge current:

$$
\begin{gathered}
\mathrm{a}(\mathrm{t})=\mathrm{C}-\gamma(\mathrm{t})=\mathrm{It} \\
\mathrm{u}(\mathrm{t})=(1-\mathrm{c}) \cdot \delta(\mathrm{t})=\frac{1(1-\mathrm{c})}{\mathrm{c}} \cdot \frac{1-\mathrm{e}^{-\mathrm{k}^{\prime} \mathrm{t}}}{\mathrm{k}^{\prime}}
\end{gathered}
$$

The first-order expansion of Eq. 39 yields:

$$
u(t)=2 I \cdot \frac{1-e^{-\beta^{2} t}}{\beta^{2}}
$$

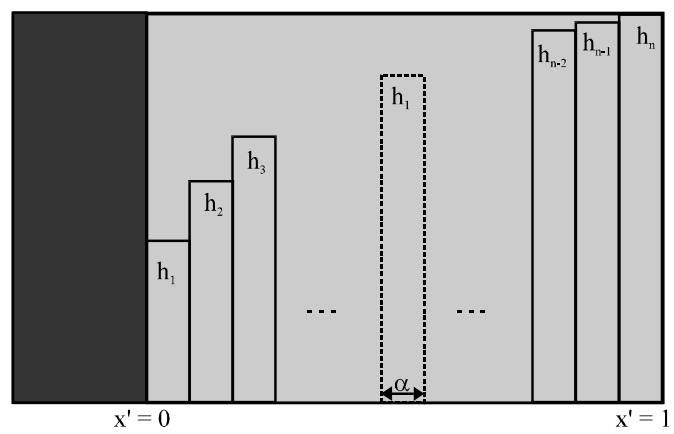

Fig. 7: Discretised diffusion model

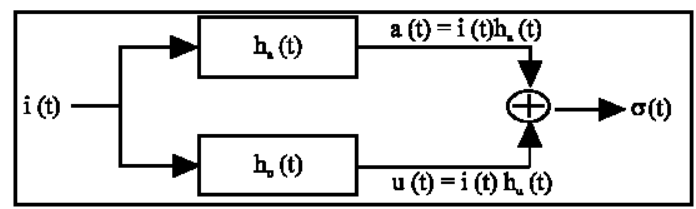

Fig. 8: Linear time-invariant system of a battery

Comparing Eq. 54 and $55, \mathrm{c}=1 / 3$ and $\mathrm{k}^{\prime}=\beta^{2}$ thus, the $\mathrm{KiBaM}$ is an approximation of the first-order diffusion model under this condition.

Frequency response: An analysis of the frequency response of both the Kinetic Battery Model and diffusion model (Rao et al., 2005) is represented by the Linear Time Invariant (LTI) system shown in Fig. 8. For both battery models, $h_{a}(t)$ is the unit step function. For the KiBaM, $h_{u}$ $(\mathrm{t})$ is given by:

$$
h_{u}(t)=\frac{1-c}{c} e^{-k^{\prime} t}
$$

while for diffusion model it is:

$$
h_{u}(t)=2 \sum_{m=0}^{\infty} e^{-\beta^{2} m^{2} t}
$$

Thus only $h_{u}(t)$ depends on the battery parameters. Therefore, Fourier transform of $h_{u}(t), h_{u}(f)$ can be used to characterize the frequency response of the battery. The results of the analysis shows that the diffusion model has a higher frequency response for high frequencies. This is due to the higher-order terms that are included in the diffusion model and not in the KiBaM.

However, both models are highly insensitive to high frequency current switching, in the ideal case the frequency response is zero for all frequencies. The insensitivity implies that currents varying faster than $0.01 \mathrm{~Hz}$ can be replaced with an average current without giving significant errors in the battery lifetime 
computations. Therefore, it is not useful to schedule tasks at small time scales smaller than minutes in order to benefit from the recovery effect, since the average current will stay the same. However, scheduling on a larger time scale, minutes or longer can be beneficial.

The frequency response is mainly determined by the size of the recovery parameter $\left(\mathrm{k}^{\prime}\right.$ or $\beta$ ) which when increased, the recovery will be faster and the battery behaviour will be closer to that of an ideal battery.

The variation of lifetime with Depth of Discharge DOD is given as (Mcdonald, 1981):

$$
\mathrm{L}=\mathrm{L}_{0} \mathrm{e}^{\mathrm{mDOD}}
$$

Where:

$\mathrm{L}=$ The number of cycles in life at DOD

$\mathrm{L}_{0}=$ The number of cycles in life obtained by extrapolating cycle-life data to zero DOD

$\mathrm{m}=$ The slope plot of $\ln \mathrm{L} / \mathrm{L}_{0}$ versus DOD

Analysis of experimental data for each battery leads to the determination of $\mathrm{L}_{0}$ and $\mathrm{m}$. Equation 58 is varified by logarithmic plot of DOD versus cycles-to-failure for varies batteries (Lindel, 1984).

Energy control characteristics of battery: The main principle for designing high-power batteries is to consider that these batteries can discharge a high percentage of their stored energy in a short period of time. In contrast, lower-power batteries can deliver more energy but only if they are discharged during a long period of time as described by Peukert law which expresses the capacity of a battery in terms of rate at which it is discharged:

$$
\mathrm{C}_{\mathrm{p}}=\mathrm{I}^{\mathrm{N}} \mathrm{t}
$$

Where:

$\mathrm{C}_{\mathrm{p}}=$ The nominal capacity $(\mathrm{Ah})$

$\mathrm{t}=$ The time of discharge $(\mathrm{h})$

$\mathrm{I}=$ The discharge current (A)

$\mathrm{N}=$ The Peukert constant which is one for an ideal battery but varies typically between 1.1 and 1.2 for the Lead-acid batteries

Besides the reduction in remaining capacity, the reduction of lifetime is significant when the battery system operates under high fluctuations. Therefore, additional measures should be taken in order to apply the battery in an efficient way as to prolong its lifetime. The best way is to split the energy and power needs into two separate components with Lead-acid batteries used as energy suppliers to balance the average energy demand while Super Capacitors or Flywheels used as power suppliers.
In this study, the use of Super Capacitors are applied to meet the fast changing power demand. A Super Capacitor is an electrochemical capacitor that has an unsually large amount of storage energy capability relative to its size when compared to common capacitors. Their advantages in relation to batteries are (Van Voorden, 2008):

- Low toxicity of material used

- Light weight

- Little degradation over hundreds of thousands of cycle

- Good reversibility

- High cycle efficiency above $95 \%$

- Very high rates of charge and discharge

However, the amount of energy stored per unit weight is considerably lower than that of electrochemical battery and the voltage varies with the energy stored. Thus sophisticated electronic control and switching equipment is required. The energy content of a super capacitor is proportional to the square of the voltage given as:

$$
\mathrm{W}_{\mathrm{c}}=\frac{1}{2} \mathrm{CV}_{\mathrm{C}}^{2}
$$

State of Charge (SOC): The state of charge greatly influences the performance of battery. Open circuit voltage decreases (approximately linearly) with increasing SOC while the internal resistance decreases slightly with increasing $\mathrm{SOC}$ at very low levels (with increasing $\mathrm{SOC}$ (Valvo et al., 1996) while the internal resistance decreases slightly with increasing SOC at very low levels $(<0.3$ full $\mathrm{SOC}$ ) then remains relatively constant until the $\mathrm{SOC}$ approaches its full state ( $>0.6$ full $\mathrm{SOC}$ ), at this point it increases rapidly.

Charging and discharge control strategies: If the output electrical power is excessive for the consumption of the loads (from period $t_{1}-t_{2}$ and $t_{7}-t_{8}$ ), the surplus is provided to charge the battery bank as shown in Fig. 9.

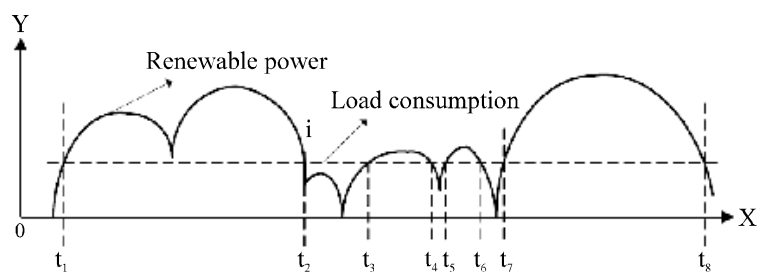

Fig. 9: Relationship between renewable power and load consumption 
Configuration: The Lead-acid Super Capacitor storage facility can be arranged in two basic configurations: DCsystem and $\mathrm{AC}$-system; the $\mathrm{AC}$-system shown in Fig. 10 is chosen due to:

- Better and simpler control of the energy exchange

- Better performance

A DC/AC converter has a limited voltage range at its $\mathrm{DC}$ side for a specific AC voltage. In standard converters of $400 \mathrm{~V}$ the DC-voltage may vary between $600-740 \mathrm{~V}$ (with the reference voltage of the system set to $670 \mathrm{~V}$ ), this limits the usable energy content of Super Capacitors however, due to its quadratic dependency most of the energy is available at the high energy regions. The control system of Fig. 11 is developed to model the SC-buffer for fast power fluctuation and battery-buffer slow energy changes of the RES.

Control strategies: It is extremely difficult to determine whether the battery should be charged or to prevent it from being charged over insufficient charge based on certain traditional mathematical models, so system based on emperical rules may be more effective. Figure 11 shows the design strategy for controlling the charging and discharging currents where the control functions to be determined mathematically.

As shown in Fig. 10, the battery supplies the energy (average power), while the Super Capacitor supplies the power deviation from the mean value. When balancing power is needed, it will be delivered by $\mathrm{SC}$ which causes SC-buffer to drop, while the battery-buffer will provide power proportional to the difference between the actual $\mathrm{SC}$ and its reference. On the other hand energy generation is greater than the demand power both SC and the battery will be charged. The four input variable for the control system of Fig. 11 are:

$$
\begin{gathered}
\Delta \mathrm{P}=\mathrm{P}-\mathrm{P}_{\text {load }} \\
\Delta \mathrm{P}^{\prime}=\frac{\mathrm{d} \Delta \mathrm{P}}{\mathrm{dt}} \\
\Delta \mathrm{T}=\mathrm{T}_{\text {load }}-\mathrm{T} \\
\Delta \mathrm{T}^{\prime}=\frac{\mathrm{d} \Delta \mathrm{T}}{\mathrm{dt}}
\end{gathered}
$$

Where:

$\Delta \mathrm{P}=$ The difference of RES power $\mathrm{P}$ and the load consumption $\mathrm{P}_{\text {load }}$
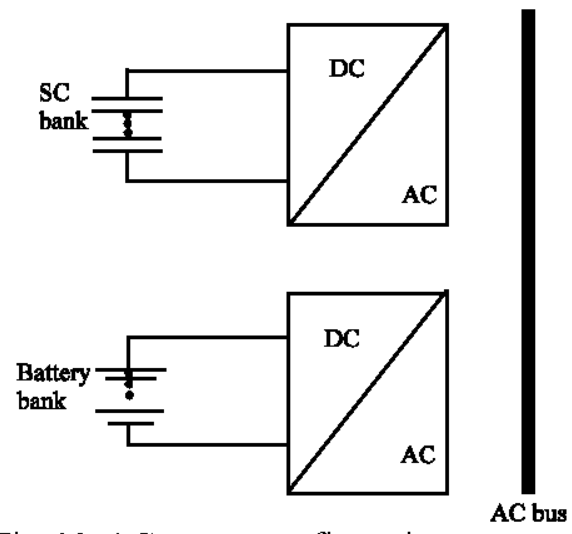

Fig. 10: AC system configuration

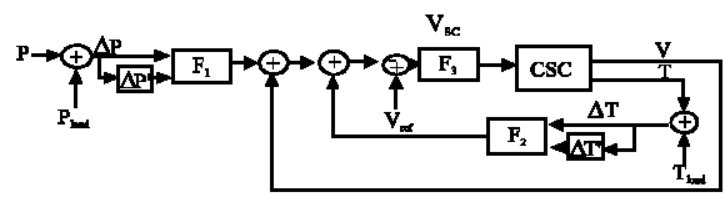

Fig. 11: Charging and discharging design control strategy

$\Delta \mathrm{P}^{\prime}=$ The rate of $\Delta \mathrm{P}$

$\Delta \mathrm{T}=$ The relative temperature of the battery $\mathrm{T}_{\text {load }}$ to the normal indoor temperature $\mathrm{T}$

The output variable is the charging voltage $\mathrm{V}$, thus the control function can be described as:

$$
\mathrm{V}=\mathrm{F}\left(\Delta \mathrm{P}, \Delta \mathrm{P}^{\prime}, \Delta \mathrm{T}, \Delta \mathrm{T}^{\prime}\right)
$$

Applying fuzzy logic to Eq. 65 with two separate parts gives (Yin et al., 2008):

$$
\begin{gathered}
\mathrm{V}_{1}=\mathrm{F}_{1}\left(\Delta \mathrm{P}, \Delta \mathrm{P}^{\prime}\right) \\
\mathrm{V}_{2}=\mathrm{F}_{2}\left(\Delta \mathrm{T}, \Delta \mathrm{T}^{\prime}\right) \\
\mathrm{V}_{1} \otimes \mathrm{V}_{2}=\mathrm{V}
\end{gathered}
$$

If the SC voltage is assumed to vary between the maximum and minimum voltage of the $400 \mathrm{~V}$ converter, Eq. 60 becomes:

$$
\mathrm{C}=2 \mathrm{E} /\left(\mathrm{V}_{\downarrow} \max ^{\uparrow} 2-\mathrm{V}_{\downarrow} \min ^{\uparrow} 2\right)
$$

\section{CONCLUSION}

The analytic models are best suited to be used in performance modeling. The analysis of the $\mathrm{KiBaM}$ and diffusion model shows that the KiBaM is actually a first order approximation of the diffusion model. Battery efficiency is modeled based on SOC and the lifetime of the 
battery depends on DOD. Batteries are not appropriate to follow sharp fluctuations (surge) in current as their lifetime are negatively affected, thus by splitting the total energy demand on the system the energy and power request can be fulfilled using super capacitors: to responde to fast changing power demand and Lead-acid battery: to account for the average energy demand. The larger the super capacitor the lower is the impact on the lifetime of the battries.

\section{REFERENCES}

Chiasserini, C. and R. Rao, 1999. Pulsed battery discharge in communication devices. Proceedings of the 5th International Conference on Mobile Computing and Networking, Aug. 15-19, Seattle, Washington, USA., pp: 88-95.

Chiasserini, C.F. and R.R. Rao, 2001a. Energy efficient battery management. IEEE J. Selected Areas Commun., 19: 1235-1245.

Chiasserini, C.F. and R.R. Rao, 2001b. Improving battery performance by using traffic shaping techniques. IEEE J. Selected Areas Commun., 19: 1385-1394.

Cloth, L., B.R. Haverkort and M.R. Jongerden, 2007. Computing battery lifetime distributions. Proceedings of the 37th Annual IEEE/IFIP International Conference on Dependable Systems and Networks, (DSN'07), IEEE Computer Society Press, pp: 780-789.

Dobner, D.J. and E.J. Woods, 1982. An Electric Vehicle Dynamic Simulation. GM Research Laboratories, USA., pp: 103-115.

Doyle, M., T.F. Fuller and J. Newman, 1993. Modeling of galvanostatic charge and discharge of the lithium/polymer/insertion cell. J. Electrochem. Soc., 140: 1526-1533.

Fuller, T.F., M. Doyle and J. Newman, 1994. Simulationand optimization of the dual lithium ion insertion cell. J. Electrochem. Soc., 141: 1-10.

Gold, S., 1997. A PSpice macromodel for lithium-ion batteries. Proceedings of 12th Annual Battery Conference on Applications and Advances, (BCAA'97), Long Beach, CA, USA., pp: 215-222.

Hageman, S.C., 1993. Simple PSpice models let you simulate common battery types. Electronic Des. News, 38: 117-129.

Jongerden, M.R. and B.R. Haverkort, 2008. Battery modeling. Centre for Telematics and Information Technology, University of Twente, Technical Report. http://eprints.eemcs.utwente.nl/11645/.

Lindel, D., 1984. Handbook of Batteries and Fuel Cells. McGraw-Hill, New York, pp: 13-19.
Manwell, J. and J. McGowan, 1993. Lead acid battery storage model for hybrid energy systems. Solar Energy, 50: 399-405.

Manwell, J., B.E. McGowan and A. Leotta, 1994. Evaluation of battery models for wind/hybrid power system simulation. Proceedings of the 5th European Wind Energy Association Conference, (EWAEC'94), Thessaloniki, pp: 1182-1187.

McDonald, A.T., 1981. Reducing battery costs for electric vehicles through optimal depth-of-discharge. Proceedings of the 4th EVC Symposium.

Moore, S. and E. Merhdad, 1996. An emperically based electrosource horizon lead-acid battery model. Strategies in Electric and Hybrid Vehicle Design, SP1156, Texas A and M, pp: 135-138.

Oneyama, N., 1998. Air compressor and its operation control. J. Energy Conserv. Centre, 50: 65-73.

Rakhmatov, D. and S. Vrudhula, 2001. An analytical highlevel battery model for use in energy management of portable electronic systems. Proceedings of the International Conference on Computer Aided Design, Nov. 4-8, San Jose, California, pp: 488-493.

Rao, R., S.B.K. Vrudhula and N. Chang, 2005. Battery optimization vs energy optimization: Which to choose and when?. Proceedings of the International Conference on Computer Aided Design, Nov. 6-10, IEEE Computer Society, pp: 439-445.

Roan, V.P. and R. Anand, 1993. An Approach to Incorporating Age and Electrolytic Temperature Effects on the Performance Simulation of Electric/Hybrid Vehicle Batteries. American Chemical Society, University of Florida, pp: 2229- 2237.

Unnewehr, L.E. and S.A. Nasar, 1982. Electric Vehicle Technology. John Wiley, New York, pp: 81-91.

Valvo, M., F.E. Wicks, D. Robertson and S. Rudin, 1996. Development and application of an improved equivalent circuit model of a lead acid battery. Intersociety Energy Conversion Eng. Conf., 2: 1159-1163.

Van Voorden, M.A., 2008. Power Balancing in Autonomous Renewable Energy Systems. Delft University of Technology, Drukkerij Verloop, Alblasserdam, Netherlands, pp: 148-160.

Yin, Y., X. Luo, S. Guo, Z. Zhou and J. Wang, 2008. A battery charging control strategy for renewable energy generating systems. Proceedings of the World Congress on Engineering, July 2-4, London, UK., pp: 356-361.

Zhao, Q., 2006. Integrated control of load power tracking and battery charging for stand-alone small scale wind power generating system. Master's Thesis, University of Inner Mongolia. 